\title{
NEUROBEHAVIOR AND MRI IN 22q13.3 DELETION SYNDROME
}

Neuromotor, sensory, language, communication and social development, and cerebral MRI and PET studies were performed in 8 children with 22 q13.3 deletion syndrome, at the National Institutes of Health, Necker-Enfants Malades Hospital, and other centers in Paris, France. A common developmental profile was characterized by hypotonia, sleep disorders, excessive crying, poor response to the environment suggestive but not diagnostic of autism, expressive language delay, sensory processing and neuromotor disorders. Cognitive tests revealed mild-to-severe delay in all developmental milestones, verbal and imitation more than motor skills. Episodic symptoms prompting an EEG included acute hypotonia, repetitive rolling of tongue, eyelid flutter, vagal syncope, and standing still at attention. One had bifrontal spikes but none had epilepsy. Brain MRI was normal or showed a thin corpus callosum, and PET studies identified a localized dysfunction of the left temporal lobe and hypoperfusion in the amygdala, as compared to a group of mentally retarded control children. This description of an underdiagnosed syndrome should lead to more frequent recognition. (Philippe A, Boddaert N, Vaivre-Douret L, et al. Neurobehavioral profile and brain imaging study of the 22q13.3 deletion syndrome in childhood. Pediatrics August 2008;122:e376e382). (Respond: Anne Philippe MD, PhD, INSERM U781, Hopital Necker-Enfants Malades, 149 Rue de Sevres, 75015 Paris, France. E-mail: anne.philippe(anecker.fr).

COMMENT. The neurobehavioral description of the $22 \mathrm{q} 13.3$ deletion syndrome resembles that of pervasive developmental disorders but is distinct from autism. The 8 children in this study shared a common developmental course characterized by hypotonia, sensory and sleep disorders, global developmental delay, lack of emotion and inappropriate facial expression, episodic and stereotyped movements and postures. These symptoms should prompt a chromosome analysis with special attention to the 22 qter deletion.

\section{SEIZURE DISORDERS}

\section{KETOGENIC DIET FOR EPILEPSY AND FOCAL MALFORMATION}

The efficacy and long-term treatment outcome of a classic ketogenic diet (KD) addon treatment (4:1 lipid/nonlipid ratio, without initial fasting and fluid restriction) were evaluated retrospectively in 47 children with intractable epilepsy and focal malformation of cortical development, in a study at Severance Children's and Sanggye Park Hospitals, Seoul, Korea. At 3 months after diet initiation, 21 (44.7\%) were seizure free, and $29(62 \%)$ had $>50 \%$ seizure reduction. Of 21 with complete seizure control at 3 months, 16 (76\%) continued the diet for 2 years without relapse; $10(48 \%)$ remained seizure-free after discontinuing the diet, at mean follow-up of 3 years 10 months. Diet was discontinued in 2 patients who developed hemorrhagic gastritis, and diet intolerance occurred in 5 patients. Of 19 patients whose seizures were not completely controlled during the KD and in 3 who had recurrences after diet withdrawal, $13(59 \%)$ became seizure-free after undergoing epilepsy surgery. All patients were followed for at least 12 months after completion of the KD. In the management of intractable seizures due to focal cortical maldevelopment, patients who 